\title{
Integrated analysis of a IncRNA-mRNA network reveals a potential mechanism underlying necrotizing enterocolitis
}

\author{
WENJUAN CHEN ${ }^{1,2^{*}}$, XIANGYUN YAN $^{1,2^{*}}$, TIAN TIAN ${ }^{1,3^{*}}$, RU YAN $^{1,2}$, XINGYUN WANG $^{1,2}$, \\ ZHANGBIN YU ${ }^{1,2}$, YINGZHENG LI ${ }^{1,2}$, LE ZHANG ${ }^{1,2}$ and SHUPING HAN ${ }^{1,2}$ \\ ${ }^{1}$ Department of Neonatology, Wuxi Children's Hospital Affiliated to Nanjing Medical University, \\ Wuxi, Jiangsu 214023; ${ }^{2}$ Department of Pediatrics, Women's Hospital of Nanjing Medical University \\ (Nanjing Maternity and Child Health Care Hospital), Nanjing, Jiangsu 210004; ${ }^{3}$ Department of Child Health Care, \\ The First Affiliated Hospital of Nanjing Medical University, Nanjing, Jiangsu 210029, P.R. China
}

Received August 29, 2019; Accepted March 25, 2020

DOI: $10.3892 / \mathrm{mmr} .2020 .11083$

\begin{abstract}
Previous studies have shown that long non-coding RNAs (lncRNAs) serve important roles in necrotizing enterocolitis (NEC). However, the underlying mechanisms remain largely unknown. In order to examine the potential role of lncRNAs in NEC, the present study investigated lncRNA and mRNA expression profiles in NEC lesions and adjacent intestinal tissues using Next Generation Sequencing. A total of 4,202 differentially expressed lncRNAs (fold-change $>2$; $\mathrm{P}<0.05$ ) and 7,860 differentially expressed mRNAs (fold-change $>2$; $\mathrm{P}<0.05$ ) were identified. Moreover, 5 dysregulated $\operatorname{lncRNAs}$ and $5 \mathrm{mRNAs}$ were randomly selected, and further assessed by reverse transcription-quantitative PCR in vitro. Gene Ontology and Kyoto Encyclopedia of Genes and Genomes analyses demonstrated that the differentially expressed lncRNAs were closely associated with NEC, and were enriched in 'inflammatory response', 'Toll-like receptor binding', 'PPAR signaling pathway', 'PI3K-Akt signaling pathway', 'transforming growth factor- $\beta$ signaling pathway' and 'hypoxia-inducible factor 1 signaling pathway'. In addition, co-expression analysis demonstrated that these lncRNAs, including lncRNA ENST00000623580, lncRNA NONHSAT180418.1, IncRNA NONHSAT125636.2 and
\end{abstract}

Correspondence to: Dr Le Zhang, Department of Neonatology, Wuxi Children's Hospital Affiliated to Nanjing Medical University, 111 Middle Renmin Road, Wuxi, Jiangsu 214023, P.R. China

E-mail: zhle@njmu.edu.cn

Dr Shuping Han, Department of Pediatrics, Women's Hospital of Nanjing Medical University (Nanjing Maternity and Child Health Care Hospital), 123 Tian Fei Xiang, Mo Chou Road, Nanjing, Jiangsu 210004, P.R. China

E-mail: shupinghan@njmu.edu.cn

${ }^{*}$ Contributed equally

Key words: necrotizing enterocolitis, long non-coding RNAs, co-expression network, pathways
NONHSAT087855.2, may mediate the pathogenesis and development of NEC via lncRNA-mRNA network interactions. Therefore, the present study provided a novel insight into the role of lncRNAs in NEC.

\section{Introduction}

Necrotizing enterocolitis (NEC) is an acute life-threatening gastrointestinal disease that predominantly affects premature neonates $(1,2)$. The morbidity of NEC is rising rapidly with the continuing increased rate of survival of preterm infants (3). Patients that survive severe NEC may develop serious sequelae, including gastrointestinal complications and abnormal neurodevelopment (4). Multiple factors contribute to NEC, including premature delivery, intestinal bacterial colonization and infant formula feeding (5). Furthermore, NEC is characterized by severe intestinal inflammation, impaired mucosal healing and intestinal necrosis (4). However, the etiology and pathogenesis of NEC are not fully understood.

Long non-coding RNAs (lncRNAs) are autonomously transcribed non-coding RNAs, which are $>200$ nucleotides in length (6). IncRNAs can act at the transcriptional, post-transcriptional and epigenetic levels via cis-or trans-acting pathways $(7,8)$. Previous studies have reported the potential importance of lncRNAs in maintaining the intestinal barrier and mediating inflammatory lesions (9-11). For example, lncRNA Uc.173 was revealed to enhance intestinal epithelial barrier function by increasing the expression of claudin 1 (9). However, lncRNA Uc.173 silencing leads to dysfunction of the intestinal epithelial barrier in vitro (9). Another study showed that lncRNA SPRY4-intronic transcript 1 (SPRY4-IT1) regulates the intestinal epithelial barrier by affecting the expression levels of claudin-1, junctional adhesion molecule 1 and occludin (10). Moreover, lncRNA SPRY4-IT1 overexpression in intestinal mucosa in mice protects the intestinal barrier against infection (10). In addition, IncRNA H19 promotes the proliferation of intestinal epithelial cells by directly binding to tumor protein p53 protein, microRNA34a and let-7 (11). Therefore, these previous results indicated that lncRNAs directly affect the expression of mRNAs via co-expression networks. However, 
the role of lncRNA-mRNA networks in the pathogenesis of NEC is not fully understood. Thus, the construction of such networks may increase the understanding of the mechanisms underlying NEC.

In the present study, the expression profiles of lncRNAs and mRNAs in NEC lesion and adjacent intestinal tissues obtained from patients with NEC were analyzed by Next Generation Sequencing (NGS). Additionally, a number of these genes were validated by reverse transcription-quantitative PCR (RT-qPCR) in vitro. Moreover, Gene Ontology (GO) and Kyoto Encyclopedia of Genes and Genomes (KEGG) analyses were performed to assess the potential functions of the differentially expressed lncRNAs and mRNAs. In addition, a lncRNA-mRNA network was constructed in order to identify the potential underlying mechanism of NEC. The present results suggested that lncRNAs in intestinal tissues may serve an important role in the development of NEC.

\section{Materials and methods}

Sample collection and ethics statement. Preterm infants (25-36 weeks of age; one female and two males) with stage III acute NEC undergoing bowel resection were enrolled in the present study between October 2017 and October 2018 at The Affiliated Wuxi Children's Hospital of Nanjing Medical University. The diagnosis of NEC was based on Bell's stages (12). Infants with other serious intestinal diseases, such as congenital megacolon, were excluded. Tissues of the small bowel segment with NEC lesions that exhibited perforation or necrosis, and adjacent healthy tissue [referred to as the control (CTL)] were resected and stored at $-80^{\circ} \mathrm{C}$ in liquid nitrogen until analysis. The study protocol was approved by the Institutional Review Board of The Affiliated Wuxi Children's Hospital of Nanjing Medical University (approval no. 2017-EYLL-115). Informed consent was obtained from the parents or guardians of all patients.

RNA isolation, quality control and RNA-Seq. Total RNA was extracted from the tissues using a mirVana microRNA isolation kit (cat. no. AM1561; Ambion; Thermo Fisher Scientific, Inc.) according to the manufacturer's protocol. RNA integrity was subsequently assessed using an Agilent Bioanalyzer 2100 system (Agilent Technologies, Inc.). RNA was further purified using an RNAClean XP kit (Beckman Coulter, Inc.) and an RNase-Free DNase set (Qiagen $\mathrm{GmbH}$ ). The samples were then subjected to NGS. The mRNAs and IncRNAs were identified using an Illumina platform (HiSeq 2500; Illumina, Inc.). The gene abundance was calculated using StringTie (version no. 1.3.0) (13). NGS, data acquisition and processing were performed by Shanghai Biotechnology Corporation.

NEC cell model construction. The human normal colorectal FHC cell line (American Type Culture Collection) was cultured in RPMI-1640 medium (Gibco; Thermo Fisher Scientific, Inc.) containing 5\% FBS (Gibco; Thermo Fisher Scientific, Inc.) and $1 \%$ penicillin/streptomycin solution at $37^{\circ} \mathrm{C}$ and $5 \% \mathrm{CO}_{2}$. In order to establish the NEC cell model, FHC cells were treated with $200 \mu \mathrm{g} / \mathrm{ml}$ lipopolysaccharide (LPS; Sigma-Aldrich; Merck KGaA) at $37^{\circ} \mathrm{C}$ for $6 \mathrm{~h}$, referring to the dose and time of LPS treatment described in previous study (14). LPS used in the present study was obtained from Escherichia coli O111:B4.

$R N A$ preparation and $R T-q P C R$. A total of $5 \mathrm{mRNAs}$ and 5 lncRNAs were randomly selected and further assessed by RT-qPCR. Total RNA from FHC cells was extracted using TRIzol ${ }^{\circledR}$ reagent (Thermo Fisher Scientific, Inc.) according to the manufacturer's instructions. RT was performed using a PrimeScript RT reagent kit (Takara Bio, Inc.) according to the manufacturer's instructions $\left(37^{\circ} \mathrm{C}\right.$ for $15 \mathrm{~min} ; 85^{\circ} \mathrm{C}$ for $5 \mathrm{sec}$; $4^{\circ} \mathrm{C}$ for $10 \mathrm{~min}$ ). qPCR was subsequently performed using a SYBR Green PCR kit (Takara Bio, Inc.). The following thermocycling conditions were used: $95^{\circ} \mathrm{C}$ for $10 \mathrm{~min} ; 95^{\circ} \mathrm{C}$ for $10 \mathrm{sec}, 60^{\circ} \mathrm{C}$ for $30 \mathrm{sec}, 40$ cycles. The primer pairs used for qPCR are presented in Table SI. Gene expression was calculated using the $2^{-\Delta \Delta \mathrm{Cq}}$ method (15), and normalized to the expression of the internal reference gene GAPDH. Data are presented as the average of 3 independent experiments.

Protein extraction and western blotting. Total protein from FHC cells was extracted using RIPA lysis buffer (Beyotime Institute of Biotechnology) containing $1 \%$ phenylmethylsulfonyl fluoride. The protein concentration was measured by a BCA assay kit (Thermo Fisher Scientific, Inc.). A total of $20 \mu \mathrm{g}$ protein/lane was separated via SDS-PAGE on a $10 \%$ gel and transferred onto a PVDF membrane. The membrane was subsequently blocked with $5 \%$ skimmed milk for $1 \mathrm{~h}$ at room temperature and incubated with primary antibodies against Toll-like receptor-4 (TLR4; 1:1,000; cat. no. bs-20594R; BIOSS) and $\beta$-actin $(1: 1,000$; cat. no. CST-4970; Cell Signaling Technology, Inc.) for $12 \mathrm{~h}$ at $4^{\circ} \mathrm{C}$. Following primary antibody incubation, the membrane was incubated with a horseradish peroxidase conjugated-secondary antibody (1:5,000; cat. no. CST-7074; Cell Signaling Technology, Inc.) for $30 \mathrm{~min}$ at room temperature. Proteins were visualized using an enhanced chemiluminescence detection system (Pierce ECL Plus Western Blotting Substrate; Thermo Fisher Scientific, Inc.). Protein bands were analyzed by ImageJ software (version 1.47; National Institutes of Health).

Hematoxylin and eosin staining. Intestines were fixed in $4 \%$ paraformaldehyde overnight at room temperature, dehydrated with various concentrations of ethanol $(50,75,85,95$ and $100 \%$ ethanol) and xylene, and embedded in paraffin. Paraffin-embedded small intestinal specimens were cut into $5-\mu \mathrm{m}$ sections. Sections were then deparaffinized and rehydrated. Following which, the sections were stained with Mayer's hematoxylin for $30 \mathrm{sec}$ and $1 \%$ eosin Y solution for 10-30 sec at room temperature. After dehydration and mounting, the sections were examined under a light microscope, at magnification, $\mathrm{x} 200$, and images were taken.

GO and KEGG analysis. GO (version 14; www.geneontology. org) was used to analyze the biological processes, cellular components and molecular functions of the differentially expressed mRNAs and host genes of the IncRNAs. In addition, KEGG (version 4.0; www.genome.jp/kegg) pathway analysis was performed to investigate the associated pathways. GO terms and KEGG pathways were ranked by the enrichment factor. 
A

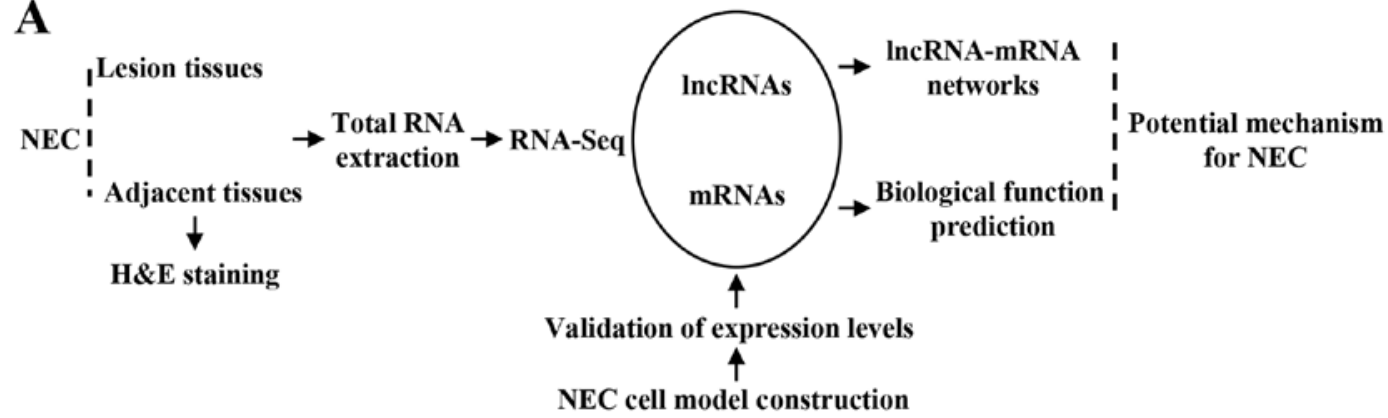

B
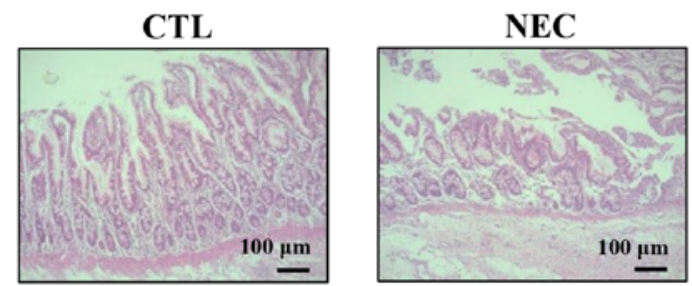

Figure 1. Analysis process. (A) NEC and adjacent healthy tissues were collected. Total RNA was subsequently extracted and a cDNA library for sequencing was established. Differentially expressed mRNAs and lncRNAs were identified and analyzed using a bioinformatics approach. (B) Intestinal tissues obtained from patients with NEC were subjected to H\&E staining. Scale bar, $100 \mu \mathrm{m}$. NEC, necrotizing enterocolitis; lncRNA, long non-coding RNA; CTL, control; $\mathrm{H} \& \mathrm{E}$, hematoxylin and eosin.

lncRNA-mRNA co-expression network. A lncRNA-mRNA network was constructed using Cytoscape software (version 3.4.0; https://cytoscape.org/). A total of 38 differentially expressed mRNAs and 44 lncRNAs, whose target genes are same to those mRNAs, were selected. In total, 80 co-expression relationships were identified.

Statistical analysis. Statistical differences were evaluated using the two independent samples t-test. Data are presented as the mean \pm standard deviation. The edgeR software package (version 3.28.1) (16) was used to identify differentially expressed lncRNAs and mRNAs, between and NEC and CTL tissues. $\mathrm{P}<0.05$ was considered to indicate a statically significant difference.

\section{Results}

Detection of IncRNA and mRNA profiles in NEC tissues. NGS was used to detect the expression of mRNAs and lncRNAs in NEC and CTL tissues. Moreover, differentially expressed mRNAs and lncRNAs were analyzed using a lncRNA-mRNA co-expression network (Fig. 1A). Furthermore, hematoxylin and eosin staining identified a disrupted mucosal architecture in NEC tissues (Fig. 1B).

Differentially expressed gene analysis. A total of 4,202 lncRNAs were significantly differentially expressed between NEC and CTL tissues (fold-change $>2 ; \mathrm{P}<0.05$ ), which included 1,657 upregulated lncRNAs and 2,545 downregulated lncRNAs (Fig. 2A; Table SII). In addition, it was identified that $7,860 \mathrm{mRNAs}$ were significantly differentially expressed between NEC and CTL tissues (fold-change $>2$; $\mathrm{P}<0.05)$, which included 4,241 upregulated mRNAs and 3,619 downregulated mRNAs (Fig. 2B; Table SIII). The top 50 significantly downregulated and upregulated lncRNAs and mRNAs are presented in Tables I and II, respectively.
Construction of the NEC cell model and validation of genes by $R T-q P C R$ in vitro. The pathology underlying NEC development requires the activation of the innate immune TLR4 on enterocytes (17). Moreover, TLR4 activation by bacterial LPS inhibits enterocyte proliferation and leads to disease progression $(18,19)$. It was demonstrated that LPS exposure increased the protein expression of TLR4 in FHCs, thus suggesting that it is a reliable cell model for studying NEC in vitro (Fig. $3 \mathrm{~A}$ ). In total, $5 \mathrm{mRNAs}$ and 5 lncRNAs were randomly selected to assess the NGS results by RT-qPCR. The present results indicated that the mRNA expression levels of interleukin (IL)-6 and IL-1 $\beta$ were upregulated, whereas the expression levels of transmembrane $4 \mathrm{~L}$ six family member 20 , solute carrier family 5 member 12 and leucine rich repeat containing 19 were downregulated (Fig. 3B-F). Furthermore, NONHSAT022134.2 was downregulated, while NONHSAT076868.2, NONHSAT159417.1, NONHSAT094312.2 and NONHSAT142596.2 were upregulated (Fig. 3G-K). Therefore, the RT-qPCR results were consistent with the NGS results.

Differentially expressed IncRNAs are associated with the inflammatory response and the transforming growth factor- $\beta$ (TGF- $\beta$ ) signaling pathway. GO and KEGG analyses of the differentially expressed lncRNAs were performed. It was identified that the terms 'intestinal absorption', 'IL-18 production', 'apoptotic process involved in morphogenesis' and 'inflammatory response' were highly enriched in the biological process category (Fig. 4A). In addition, 'immunological synapse', 'actin filament', 'cell-cell contact zone' and 'receptor' were highly enriched in the cellular component category (Fig. 4B). Furthermore, it was demonstrated that 'extracellular ATP-gated cation channel activity', 'chemorepellent activity', 'PI3K activity' and 'cAMP binding' were highly enriched in the molecular function category (Fig. 4C). The KEGG pathway analysis results identified that the 
Table I. Top 50 significantly upregulated and downregulated long non-coding RNAs.

A, Upregulated

\begin{tabular}{|c|c|c|c|}
\hline lncRNA_ID & $\log 2 \mathrm{FC}$ & P-value & Q-value \\
\hline NONHSAT155609.1 & 11.61383132 & $1.43 \times 10^{-23}$ & $1.22 \times 10^{-20}$ \\
\hline ENST00000602813 & 11.55252438 & $1.30 \times 10^{-09}$ & $7.94 \times 10^{-08}$ \\
\hline NONHSAT059749.2 & 10.91351071 & $3.06 \times 10^{-22}$ & $1.92 \times 10^{-19}$ \\
\hline NONHSAT118562.2 & 10.66845241 & $2.81 \times 10^{-19}$ & $1.06 \times 10^{-16}$ \\
\hline ENST00000620266 & 10.60925657 & $2.84 \times 10^{-30}$ & $5.92 \times 10^{-27}$ \\
\hline NONHSAT209475.1 & 10.52975989 & $1.54 \times 10^{-17}$ & $4.47 \times 10^{-15}$ \\
\hline NONHSAT159678.1 & 10.44685758 & $2.78 \times 10^{-17}$ & $7.58 \times 10^{-15}$ \\
\hline NONHSAT169788.1 & 10.30207377 & $8.74 \times 10^{-11}$ & $6.80 \times 10^{-09}$ \\
\hline NONHSAT123206.2 & 10.20925898 & $2.57 \times 10^{-10}$ & $1.81 \times 10^{-08}$ \\
\hline NONHSAT094312.2 & 10.08888094 & $2.95 \times 10^{-27}$ & $4.47 \times 10^{-24}$ \\
\hline NONHSAT009758.2 & 9.718282592 & $1.25 \times 10^{-14}$ & $2.10 \times 10^{-12}$ \\
\hline ENST00000499624 & 9.681004925 & $6.54 \times 10^{-25}$ & $7.20 \times 10^{-22}$ \\
\hline NONHSAT177823.1 & 9.583016227 & $1.06 \times 10^{-16}$ & $2.59 \times 10^{-14}$ \\
\hline NONHSAT159417.1 & 9.438955909 & $1.51 \times 10^{-23}$ & $1.26 \times 10^{-20}$ \\
\hline NONHSAT173659.1 & 9.328552802 & $1.54 \times 10^{-17}$ & $4.47 \times 10^{-15}$ \\
\hline NONHSAT137151.2 & 9.243165734 & $2.22 \times 10^{-08}$ & $1.04 \times 10^{-06}$ \\
\hline NONHSAT206983.1 & 9.228339375 & $2.26 \times 10^{-22}$ & $1.47 \times 10^{-19}$ \\
\hline NONHSAT178474.1 & 9.086352515 & $1.08 \times 10^{-10}$ & $8.23 \times 10^{-09}$ \\
\hline ENST00000628476 & 9.043520963 & $4.70 \times 10^{-11}$ & $3.93 \times 10^{-09}$ \\
\hline NONHSAT081637.2 & 9.023472571 & $1.02 \times 10^{-05}$ & 0.000234023 \\
\hline NONHSAT076868.2 & 8.99220343 & $1.31 \times 10^{-32}$ & $3.36 \times 10^{-29}$ \\
\hline NONHSAT155904.1 & 8.936911199 & $7.85 \times 10^{-14}$ & $1.12 \times 10^{-11}$ \\
\hline NONHSAT180405.1 & 8.90507266 & $3.21 \times 10^{-07}$ & $1.13 \times 10^{-05}$ \\
\hline NONHSAT071404.2 & 8.875787946 & $2.04 \times 10^{-20}$ & $9.59 \times 10^{-18}$ \\
\hline NONHSAT183239.1 & 8.869833121 & $3.85 \times 10^{-15}$ & $6.90 \times 10^{-13}$ \\
\hline NONHSAT083185.2 & 8.855681215 & $2.60 \times 10^{-20}$ & $1.20 \times 10^{-17}$ \\
\hline NONHSAT055522.2 & 8.835207264 & $2.66 \times 10^{-13}$ & $3.40 \times 10^{-11}$ \\
\hline ENST00000573951 & 8.78394133 & $3.14 \times 10^{-16}$ & $6.98 \times 10^{-14}$ \\
\hline NONHSAT142596.2 & 8.728768517 & $1.32 \times 10^{-19}$ & $5.25 \times 10^{-17}$ \\
\hline NONHSAT055581.2 & 8.726034495 & $2.06 \times 10^{-10}$ & $1.48 \times 10^{-08}$ \\
\hline NONHSAT050230.2 & 8.724625325 & $8.74 \times 10^{-11}$ & $6.80 \times 10^{-09}$ \\
\hline NONHSAT056662.2 & 8.670344103 & $2.25 \times 10^{-16}$ & $5.17 \times 10^{-14}$ \\
\hline ENST00000505532 & 8.58704266 & $1.64 \times 10^{-14}$ & $2.64 \times 10^{-12}$ \\
\hline NONHSAT009555.2 & 8.577747998 & $3.39 \times 10^{-06}$ & $9.05 \times 10^{-05}$ \\
\hline ENST00000635687 & 8.488271168 & $1.13 \times 10^{-18}$ & $3.91 \times 10^{-16}$ \\
\hline ENST00000523099 & 8.4516672 & $2.38 \times 10^{-06}$ & $6.72 \times 10^{-05}$ \\
\hline NONHSAT050198.2 & 8.410023146 & $1.50 \times 10^{-05}$ & 0.000327563 \\
\hline NONHSAT064287.2 & 8.405723447 & $7.03 \times 10^{-06}$ & 0.000168779 \\
\hline ENST00000558776 & 8.383710584 & 0.004874773 & 0.039194634 \\
\hline NONHSAT010340.2 & 8.380591242 & $3.29 \times 10^{-05}$ & 0.00064125 \\
\hline ENST00000441790 & 8.379233397 & $1.26 \times 10^{-07}$ & $4.90 \times 10^{-06}$ \\
\hline ENST00000426699 & 8.371776115 & $7.03 \times 10^{-06}$ & 0.000168779 \\
\hline ENST00000614899 & 8.359796918 & $1.26 \times 10^{-07}$ & $4.90 \times 10^{-06}$ \\
\hline NONHSAT182506.1 & 8.346803842 & $8.05 \times 10^{-10}$ & $5.21 \times 10^{-08}$ \\
\hline ENST00000615722 & 8.342773399 & $1.72 \times 10^{-07}$ & $6.44 \times 10^{-06}$ \\
\hline NONHSAT215498.1 & 8.331225239 & $7.54 \times 10^{-09}$ & $3.93 \times 10^{-07}$ \\
\hline NONHSAT095885.2 & 8.32856903 & $8.52 \times 10^{-07}$ & $2.65 \times 10^{-05}$ \\
\hline NONHSAT175395.1 & 8.31998397 & $1.37 \times 10^{-12}$ & $1.50 \times 10^{-10}$ \\
\hline NONHSAT108374.2 & 8.314575441 & $2.86 \times 10^{-14}$ & $4.35 \times 10-12$ \\
\hline NONHSAT207205.1 & 8.305343553 & $9.36 \times 10^{-08}$ & $3.77 \times 10^{-06}$ \\
\hline
\end{tabular}


Table I. Continued.

B, Downregulated

\begin{tabular}{|c|c|c|c|}
\hline lncRNA_ID & $\log 2 \mathrm{FC}$ & P-value & Q-value \\
\hline NONHSAT183597.1 & -16.48090468 & $8.79 \times 10^{-58}$ & $2.93 \times 10^{-53}$ \\
\hline NONHSAT168464.1 & -14.21176751 & $2.63 \times 10^{-42}$ & $2.92 \times 10^{-38}$ \\
\hline NONHSAT213758.1 & -12.54912544 & $1.45 \times 10^{-36}$ & $8.05 \times 10^{-33}$ \\
\hline NONHSAT185013.1 & -12.48955925 & $8.00 \times 10^{-31}$ & $1.90 \times 10^{-27}$ \\
\hline NONHSAT152066.1 & -12.17786195 & $5.62 \times 10^{-34}$ & $1.87 \times 10^{-30}$ \\
\hline NONHSAT024359.2 & -11.87327964 & $3.83 \times 10^{-45}$ & $6.38 \times 10^{-41}$ \\
\hline MSTRG.44916.1 & -11.60824268 & $7.79 \times 10^{-35}$ & $3.24 \times 10^{-31}$ \\
\hline NONHSAT181735.1 & -11.43001449 & $6.93 \times 10^{-27}$ & $1.00 \times 10^{-23}$ \\
\hline NONHSAT160167.1 & -11.41835274 & $3.88 \times 10^{-24}$ & $3.49 \times 10^{-21}$ \\
\hline NONHSAT165927.1 & -11.25071761 & $1.29 \times 10^{-24}$ & $1.23 \times 10^{-21}$ \\
\hline NONHSAT159286.1 & -11.10183428 & $9.99 \times 10^{-26}$ & $1.33 \times 10^{-22}$ \\
\hline NONHSAT091106.2 & -11.07224496 & $4.78 \times 10^{-28}$ & $7.97 \times 10^{-25}$ \\
\hline NONHSAT175707.1 & -11.04329687 & $1.25 \times 10^{-25}$ & $1.61 \times 10^{-22}$ \\
\hline ENST00000504773 & -10.84721244 & $4.46 \times 10^{-23}$ & $3.30 \times 10^{-20}$ \\
\hline NONHSAT091102.2 & -10.66881414 & $1.16 \times 10^{-17}$ & $3.45 \times 10^{-15}$ \\
\hline NONHSAT159894.1 & -10.62188109 & $1.57 \times 10^{-21}$ & $9.02 \times 10^{-19}$ \\
\hline NONHSAT031256.2 & -10.60250823 & $1.47 \times 10^{-37}$ & $1.22 \times 10^{-33}$ \\
\hline NONHSAT123764.2 & -10.50424036 & $8.82 \times 10^{-33}$ & $2.45 \times 10^{-29}$ \\
\hline ENST00000507311 & -10.47456391 & $1.96 \times 10^{-30}$ & $4.36 \times 10^{-27}$ \\
\hline NONHSAT171172.1 & -10.41117159 & $9.23 \times 10^{-23}$ & $6.28 \times 10^{-20}$ \\
\hline NONHSAT162645.1 & -10.33478733 & $3.39 \times 10^{-17}$ & $9.04 \times 10^{-15}$ \\
\hline NONHSAT091107.2 & -10.32292548 & $2.57 \times 10^{-23}$ & $2.04 \times 10^{-20}$ \\
\hline NONHSAT139922.2 & -10.31278929 & $5.33 \times 10^{-19}$ & $1.97 \times 10^{-16}$ \\
\hline NONHSAT183589.1 & -10.28237601 & $5.84 \times 10^{-25}$ & $6.71 \times 10^{-22}$ \\
\hline NONHSAT196167.1 & -10.27748084 & $6.07 \times 10^{-23}$ & $4.31 \times 10^{-20}$ \\
\hline MSTRG.37793.3 & -10.20152795 & $2.73 \times 10^{-22}$ & $1.75 \times 10^{-19}$ \\
\hline NONHSAT188793.1 & -10.11670026 & $1.02 \times 10^{-09}$ & $6.43 \times 10^{-08}$ \\
\hline NONHSAT152222.1 & -10.03914993 & $1.25 \times 10^{-14}$ & $2.10 \times 10^{-12}$ \\
\hline ENST00000606993 & -10.00747462 & $1.58 \times 10^{-18}$ & $5.28 \times 10^{-16}$ \\
\hline NONHSAT115736.2 & -9.985583318 & $2.27 \times 10^{-13}$ & $2.94 \times 10^{-11}$ \\
\hline NONHSAT157008.1 & -9.951490231 & $7.85 \times 10^{-14}$ & $1.12 \times 10^{-11}$ \\
\hline NONHSAT160671.1 & -9.908059683 & $3.39 \times 10^{-15}$ & $6.15 \times 10^{-13}$ \\
\hline ENST00000427901 & -9.906883277 & $4.70 \times 10^{-11}$ & $3.93 \times 10^{-09}$ \\
\hline NONHSAT192263.1 & -9.864619513 & $3.19 \times 10^{-18}$ & $1.01 \times 10^{-15}$ \\
\hline NONHSAT182621.1 & -9.857221425 & $2.57 \times 10^{-11}$ & $2.28 \times 10^{-09}$ \\
\hline NONHSAT094167.2 & -9.856028479 & $1.67 \times 10^{-13}$ & $2.24 \times 10^{-11}$ \\
\hline ENST00000419296 & -9.82059815 & $1.29 \times 10^{-24}$ & $1.23 \times 10^{-21}$ \\
\hline NONHSAT041891.2 & -9.794594044 & $2.11 \times 10^{-09}$ & $1.24 \times 10^{-07}$ \\
\hline NONHSAT176544.1 & -9.772352515 & $1.65 \times 10^{-09}$ & $9.95 \times 10^{-08}$ \\
\hline ENST00000455071 & -9.756925085 & $1.60 \times 10^{-24}$ & $1.48 \times 10^{-21}$ \\
\hline NONHSAT208752.1 & -9.667998866 & $2.92 \times 10^{-18}$ & $9.36 \times 10^{-16}$ \\
\hline NONHSAT022134.2 & -9.65276041 & $7.91 \times 10^{-37}$ & $5.27 \times 10^{-33}$ \\
\hline NONHSAT017148.2 & -9.551998808 & $3.83 \times 10^{-11}$ & $3.29 \times 10^{-09}$ \\
\hline NONHSAT017600.2 & -9.549948279 & $1.26 \times 10^{-07}$ & $4.90 \times 10^{-06}$ \\
\hline NONHSAT143306.2 & -9.53507668 & $3.95 \times 10^{-36}$ & $1.88 \times 10^{-32}$ \\
\hline NONHSAT152860.1 & -9.531346389 & $1.03 \times 10^{-18}$ & $3.63 \times 10^{-16}$ \\
\hline NONHSAT091105.2 & -9.513378928 & $1.40 \times 10^{-19}$ & $5.47 \times 10^{-17}$ \\
\hline ENST00000584749 & -9.484529301 & $8.05 \times 10^{-10}$ & $5.21 \times 10^{-08}$ \\
\hline NONHSAT060224.2 & -9.352889754 & $4.70 \times 10^{-11}$ & $3.93 \times 10^{-09}$ \\
\hline NONHSAT032678.2 & -9.348901378 & $1.43 \times 10^{-13}$ & $1.95 \times 10^{-11}$ \\
\hline
\end{tabular}

FC, fold change. 
Table II. Top 50 significantly upregulated and downregulated mRNAs.

A, Upregulated

\begin{tabular}{|c|c|c|c|c|}
\hline Gene ID & Gene name & $\log 2 \mathrm{FC}$ & P-value & Q-value \\
\hline ENSG00000228536 & RP11-392O17.1 & 9.13 & $2.33 \times 10^{-17}$ & $3.87 \times 10^{-16}$ \\
\hline ENSG00000129988 & LBP & 8.87 & $9.55 \times 10^{-30}$ & $3.12 \times 10^{-28}$ \\
\hline ENSG00000121053 & EPX & 8.64 & $1.62 \times 10^{-33}$ & $6.40 \times 10^{-32}$ \\
\hline ENSG00000267653 & RP1-193H18.3 & 8.63 & $1.30 \times 10^{-09}$ & $1.16 \times 10^{-08}$ \\
\hline ENSG00000244437 & IGKV3-15 & 8.47 & $4.11 \times 10^{-07}$ & $2.73 \times 10^{-06}$ \\
\hline ENSG00000145850 & TIMD4 & 8.34 & $7.39 \times 10^{-23}$ & $1.69 \times 10^{-21}$ \\
\hline ENSG00000259183 & MED28P6 & 8.33 & 0.001268 & 0.004697 \\
\hline ENSG00000229308 & AC010084.1 & 8.32 & $7.04 \times 10^{-08}$ & $5.18 \times 10^{-07}$ \\
\hline ENSG00000279686 & ECSCR & 8.25 & $1.97 \times 10^{-12}$ & $2.29 \times 10^{-11}$ \\
\hline ENSG00000231123 & SPATA20P1 & 8.17 & $8.35 \times 10^{-06}$ & $4.60 \times 10^{-05}$ \\
\hline ENSG00000226012 & AP001434.2 & 8.16 & 0.000667 & 0.002621 \\
\hline ENSG00000230024 & RP11-95P13.1 & 8.13 & $8.35 \times 10^{-06}$ & $4.60 \times 10^{-05}$ \\
\hline ENSG00000273153 & RP11-406H21.2 & 8.07 & 0.002421 & 0.008418 \\
\hline ENSG00000164821 & DEFA4 & 8.05 & $2.27 \times 10^{-07}$ & $1.56 \times 10^{-06}$ \\
\hline ENSG00000267436 & AC005786.7 & 8.04 & $7.45 \times 10^{-07}$ & $4.79 \times 10^{-06}$ \\
\hline ENSG00000271656 & RP11-114N19.5 & 7.99 & 0.000667 & 0.002621 \\
\hline ENSG00000211970 & IGHV4-61 & 7.93 & $2.86 \times 10^{-05}$ & 0.000146 \\
\hline ENSG00000211942 & IGHV3-13 & 7.92 & $5.33 \times 10^{-05}$ & 0.000258 \\
\hline ENSG00000259626 & MTND3P12 & 7.83 & 0.001268 & 0.004697 \\
\hline ENSG00000279903 & RP11-349F21.5 & 7.82 & $4.54 \times 10^{-06}$ & $2.60 \times 10^{-05}$ \\
\hline ENSG00000231613 & RP5-943J3.1 & 7.78 & $2.86 \times 10^{-05}$ & 0.000146 \\
\hline ENSG00000235821 & IFITM4P & 7.73 & 0.000187 & 0.00082 \\
\hline ENSG00000214402 & LCNL1 & 7.71 & $6.67 \times 10^{-48}$ & $4.38 \times 10^{-46}$ \\
\hline ENSG00000225938 & RP4-575N6.4 & 7.67 & 0.000667 & 0.002621 \\
\hline ENSG00000211946 & IGHV3-20 & 7.62 & 0.000667 & 0.002621 \\
\hline ENSG00000256039 & RP11-291B21.2 & 7.62 & $1.26 \times 10^{-07}$ & $8.98 \times 10^{-07}$ \\
\hline ENSG00000158104 & HPD & 7.55 & $2.07 \times 10^{-21}$ & $4.43 \times 10^{-20}$ \\
\hline ENSG00000235151 & AC 114730.2 & 7.51 & $5.33 \times 10^{-05}$ & 0.000258 \\
\hline ENSG00000239839 & DEFA3 & 7.51 & $8.35 \times 10^{-06}$ & $4.60 \times 10^{-05}$ \\
\hline ENSG00000275558 & RN7SKP175 & 7.49 & 0.008941 & 0.02691 \\
\hline ENSG00000239353 & RP11-492E3.51 & 7.45 & 0.002421 & 0.008418 \\
\hline ENSG00000149516 & MS4A3 & 7.45 & $2.99 \times 10^{-27}$ & $8.62 \times 10^{-26}$ \\
\hline ENSG00000243066 & RN7SL842P & 7.44 & 0.008941 & 0.02691 \\
\hline ENSG00000196415 & PRTN3 & 7.39 & $8.74 \times 10^{-55}$ & $7.16 \times 10^{-53}$ \\
\hline ENSG00000211950 & IGHV1-24 & 7.35 & 0.002421 & 0.008418 \\
\hline ENSG00000275791 & TRBV10-3 & 7.34 & 0.004643 & 0.014979 \\
\hline ENSG00000211664 & IGLV2-18 & 7.31 & 0.004643 & 0.014979 \\
\hline ENSG00000226660 & TRBV2 & 7.31 & 0.002421 & 0.008418 \\
\hline ENSG00000250579 & CTD-2297D10.2 & 7.31 & $1.36 \times 10^{-06}$ & $8.41 \times 10^{-06}$ \\
\hline ENSG00000166819 & PLIN1 & 7.31 & $4.63 \times 10^{-102}$ & $1.30 \times 10^{-99}$ \\
\hline ENSG00000273006 & RP11-314C9.2 & 7.31 & 0.017298 & 0.047391 \\
\hline ENSG00000258082 & RP11-443B7.3 & 7.31 & $4.54 \times 10^{-06}$ & $2.60 \times 10^{-05}$ \\
\hline ENSG00000240864 & IGKV1-16 & 7.30 & 0.004643 & 0.014979 \\
\hline ENSG00000279180 & RP11-417O18.1 & 7.30 & $1.36 \times 10^{-06}$ & $8.41 \times 10^{-06}$ \\
\hline ENSG00000184811 & TUSC5 & 7.26 & $2.61 \times 10^{-40}$ & $1.33 \times 10^{-38}$ \\
\hline ENSG00000234436 & AC008984.7 & 7.20 & 0.004643 & 0.014979 \\
\hline ENSG00000232286 & RP11-80K6.2 & 7.19 & 0.002421 & 0.008418 \\
\hline ENSG00000211747 & TRBV20-1 & 7.17 & 0.004643 & 0.014979 \\
\hline ENSG00000254673 & RP11-598P20.5 & 7.16 & 0.000187 & 0.00082 \\
\hline ENSG00000231863 & RP3-428L16.1 & 7.16 & 0.000667 & 0.002621 \\
\hline
\end{tabular}


Table II. Continued.

B, Downregulated

\begin{tabular}{|c|c|c|c|c|}
\hline Gene ID & Gene name & $\log 2 \mathrm{FC}$ & P-value & Q-value \\
\hline ENSG00000147676 & MAL2 & -9.98 & $2.32 \times 10^{-71}$ & $3.10 \times 10^{-69}$ \\
\hline ENSG00000140832 & MARVELD3 & -9.97 & $4.72 \times 10^{-93}$ & $1.09 \times 10^{-90}$ \\
\hline ENSG00000158125 & $\mathrm{XDH}$ & -9.97 & $1.84 \times 10^{-153}$ & $1.60 \times 10^{-150}$ \\
\hline ENSG00000272141 & RP11-465B22.8 & -9.97 & $7.32 \times 10^{-33}$ & $2.79 \times 10^{-31}$ \\
\hline ENSG00000235122 & RP3-417L20.4 & -9.96 & $2.50 \times 10^{-16}$ & $3.88 \times 10^{-15}$ \\
\hline ENSG00000234155 & RP11-30P6.6 & -9.93 & $5.74 \times 10^{-57}$ & $4.96 \times 10^{-55}$ \\
\hline ENSG00000160868 & CYP3A4 & -9.92 & $9.78 \times 10^{-159}$ & $9.35 \times 10^{-156}$ \\
\hline ENSG00000103534 & TMC5 & -9.89 & $1.11 \times 10^{-142}$ & $7.63 \times 10^{-140}$ \\
\hline ENSG00000235523 & RP11-63P12.7 & -9.86 & $9.90 \times 10^{-15}$ & $1.37 \times 10^{-13}$ \\
\hline ENSG00000165841 & CYP2C19 & -9.84 & $2.86 \times 10^{-62}$ & $2.90 \times 10^{-60}$ \\
\hline ENSG00000253313 & C1orf210 & -9.83 & $1.62 \times 10^{-47}$ & $1.05 \times 10^{-45}$ \\
\hline ENSG00000084674 & APOB & -9.82 & $1.30 \times 10^{-230}$ & $1.93 \times 10^{-226}$ \\
\hline ENSG00000118094 & TREH & -9.81 & $8.09 \times 10^{-90}$ & $1.75 \times 10^{-87}$ \\
\hline ENSG00000172016 & REG3A & -9.80 & $4.24 \times 10^{-130}$ & $2.03 \times 10^{-127}$ \\
\hline ENSG00000044012 & GUCA2B & -9.78 & $4.31 \times 10^{-20}$ & $8.54 \times 10^{-19}$ \\
\hline ENSG00000081051 & AFP & -9.77 & $4.20 \times 10^{-70}$ & $5.49 \times 10^{-68}$ \\
\hline ENSG00000169876 & MUC17 & -9.77 & $1.80 \times 10^{-195}$ & $4.11 \times 10^{-192}$ \\
\hline ENSG00000173467 & AGR3 & -9.69 & $3.30 \times 10^{-36}$ & $1.48 \times 10^{-34}$ \\
\hline ENSG00000131910 & NR0B2 & -9.68 & $8.08 \times 10^{-32}$ & $2.92 \times 10^{-30}$ \\
\hline ENSG00000122711 & SPINK4 & -9.64 & $3.00 \times 10^{-23}$ & $7.07 \times 10^{-22}$ \\
\hline ENSG00000165556 & $\mathrm{CDX} 2$ & -9.63 & $8.60 \times 10^{-110}$ & $2.83 \times 10^{-107}$ \\
\hline ENSG00000224916 & APOC4-APOC2 & -9.63 & $8.05 \times 10^{-52}$ & $6.09 \times 10^{-50}$ \\
\hline ENSG00000167183 & PRR15L & -9.53 & $3.86 \times 10^{-107}$ & $1.19 \times 10^{-104}$ \\
\hline ENSG00000232400 & RAD17P1 & -9.53 & $1.93 \times 10^{-42}$ & $1.06 \times 10^{-40}$ \\
\hline ENSG00000278505 & C17orf78 & -9.53 & $1.27 \times 10^{-54}$ & $1.04 \times 10^{-52}$ \\
\hline ENSG00000225329 & LHFPL3-AS2 & -9.53 & $7.93 \times 10^{-49}$ & $5.37 \times 10^{-47}$ \\
\hline ENSG00000143278 & F13B & -9.52 & $4.39 \times 10^{-49}$ & $3.00 \times 10^{-47}$ \\
\hline ENSG00000187664 & HAPLN4 & -9.51 & $5.32 \times 10^{-60}$ & $5.03 \times 10^{-58}$ \\
\hline ENSG00000127831 & VIL1 & -9.50 & $1.33 \times 10^{-163}$ & $1.65 \times 10^{-160}$ \\
\hline ENSG00000108242 & CYP2C18 & -9.49 & $3.21 \times 10^{-52}$ & $2.46 \times 10^{-50}$ \\
\hline ENSG00000136872 & ALDOB & -9.44 & $4.47 \times 10^{-204}$ & $1.66 \times 10^{-200}$ \\
\hline ENSG00000160182 & TFF1 & -9.38 & $6.20 \times 10^{-16}$ & $9.32 \times 10^{-15}$ \\
\hline ENSG00000105398 & SULT2A1 & -9.38 & $5.95 \times 10^{-128}$ & $2.76 \times 10^{-125}$ \\
\hline ENSG00000241388 & HNF1A-AS1 & -9.35 & $2.35 \times 10^{-62}$ & $2.40 \times 10^{-60}$ \\
\hline ENSG00000224163 & RP11-309L24.6 & -9.35 & $2.45 \times 10^{-15}$ & $3.57 \times 10^{-14}$ \\
\hline ENSG00000257084 & U47924.27 & -9.33 & $3.00 \times 10^{-10}$ & $2.85 \times 10^{-09}$ \\
\hline ENSG00000166391 & MOGAT2 & -9.28 & $4.57 \times 10^{-140}$ & $2.95 \times 10^{-137}$ \\
\hline ENSG00000140297 & GCNT3 & -9.28 & $4.25 \times 10^{-76}$ & $6.45 \times 10^{-74}$ \\
\hline ENSG00000275410 & HNF1B & -9.27 & $6.98 \times 10^{-76}$ & $1.06 \times 10^{-73}$ \\
\hline ENSG00000132437 & DDC & -9.27 & $6.68 \times 10^{-100}$ & $1.80 \times 10^{-97}$ \\
\hline ENSG00000171431 & KRT20 & -9.26 & $1.15 \times 10^{-99}$ & $3.07 \times 10^{-97}$ \\
\hline ENSG00000162460 & TMEM82 & -9.26 & $7.32 \times 10^{-33}$ & $2.79 \times 10^{-31}$ \\
\hline ENSG00000197408 & CYP2B6 & -9.25 & $1.08 \times 10^{-69}$ & $1.39 \times 10^{-67}$ \\
\hline ENSG00000249948 & GBA3 & -9.25 & $2.62 \times 10^{-99}$ & $6.81 \times 10^{-97}$ \\
\hline ENSG00000100604 & CHGA & -9.19 & $1.03 \times 10^{-130}$ & $5.09 \times 10^{-128}$ \\
\hline ENSG00000198944 & SOWAHA & -9.18 & $1.05 \times 10^{-73}$ & $1.49 \times 10^{-71}$ \\
\hline ENSG00000203858 & HSD3BP2 & -9.17 & $9.98 \times 10^{-24}$ & $2.40 \times 10^{-22}$ \\
\hline ENSG00000166869 & CHP2 & -9.17 & $4.15 \times 10^{-112}$ & $1.45 \times 10^{-109}$ \\
\hline ENSG00000060566 & CREB3L3 & -9.13 & $9.98 \times 10^{-136}$ & $5.58 \times 10^{-133}$ \\
\hline ENSG00000260934 & CTA-363E6.7 & -9.10 & $1.19 \times 10^{-12}$ & $1.40 \times 10^{-11}$ \\
\hline
\end{tabular}

FC, fold change. 

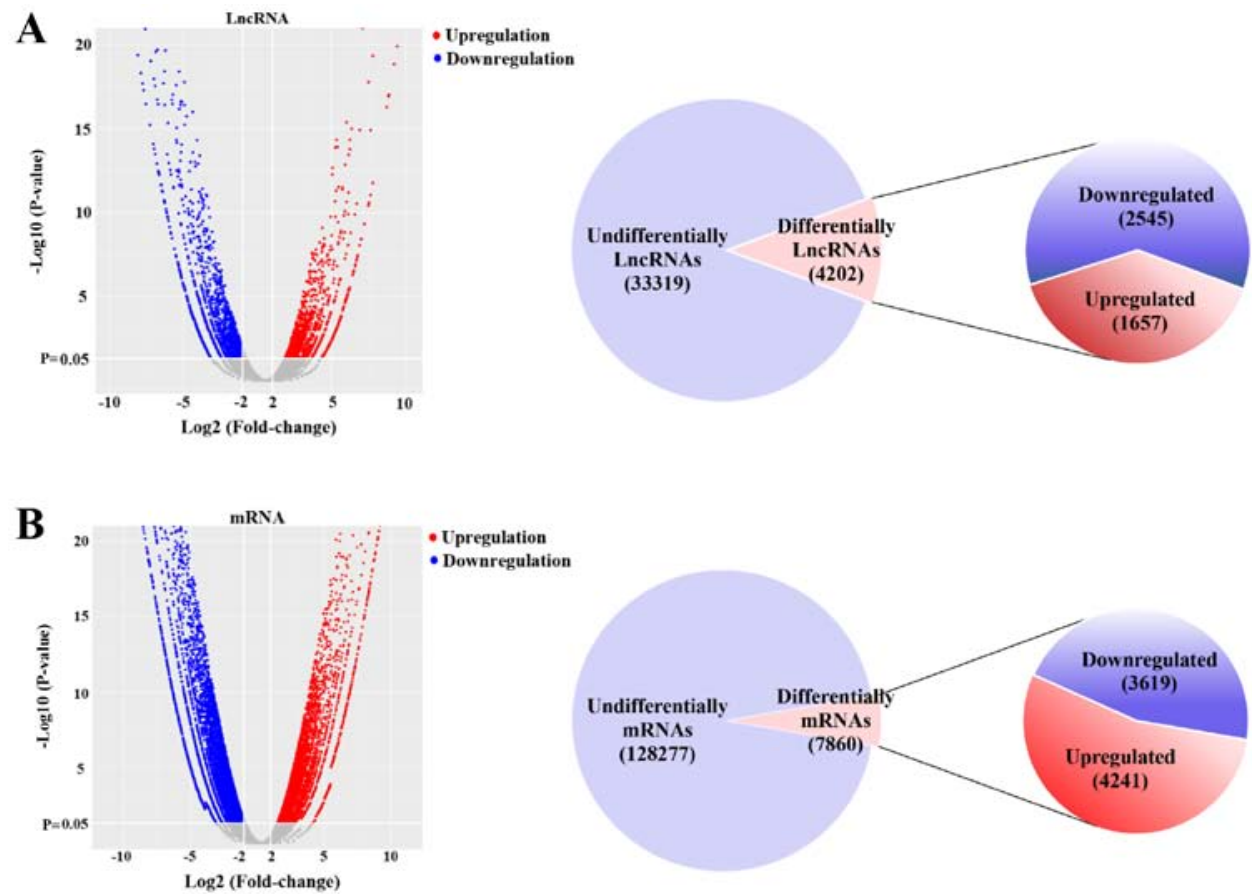

Figure 2. Differentially expressed mRNAs and lncRNAs in necrotizing enterocolitis and adjacent tissues. (A) A volcano plot was used to assess the differentially expressed lncRNAs. Of the 37,521 non-redundant lncRNAs, 4,202 lncRNAs were significantly differentially expressed (fold-change $>2$; P $<0.05$ ). A total of 1,657 and 2,545 lncRNAs were upregulated and downregulated, respectively. (B) A volcano plot was used to assess differentially expressed mRNAs. Of the 136,137 non-redundant mRNAs, 7,860 mRNAs were significantly differentially expressed (fold-change $>2 ; \mathrm{P}<0.05$ ). A total of 4,241 and 3,619 mRNAs were upregulated and downregulated, respectively. IncRNA, long non-coding RNA.
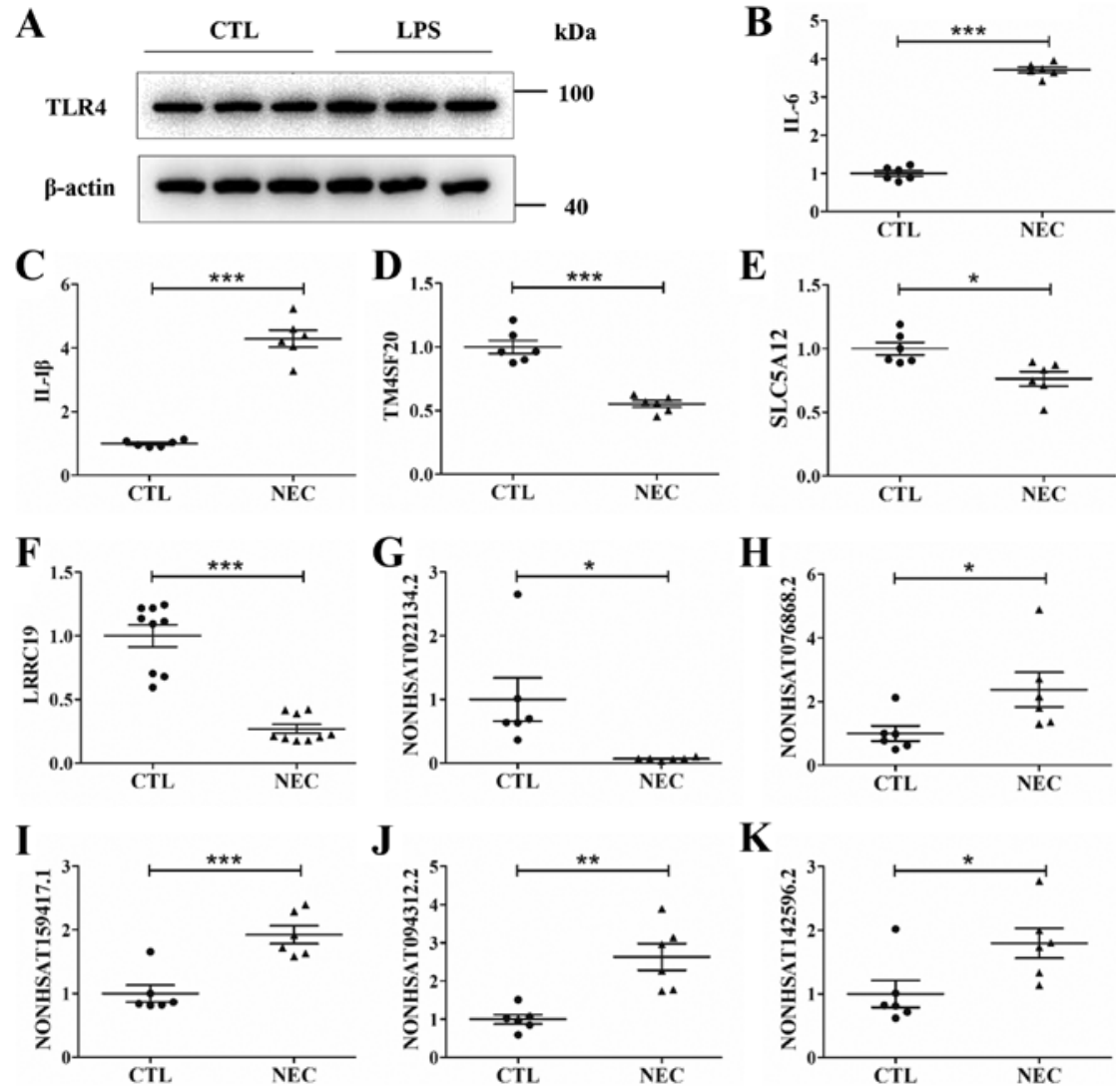

$\mathbf{E}$
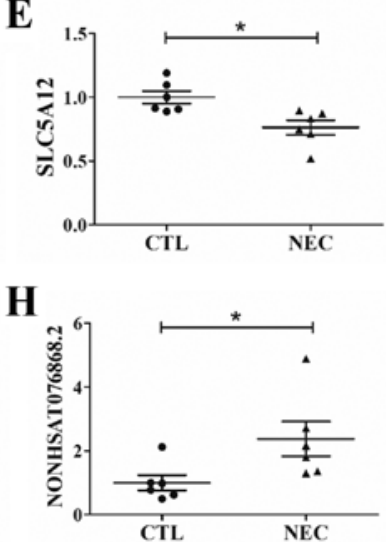

$\mathbf{K}$

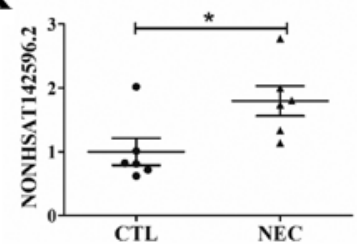

Figure 3. NEC cell model construction, and assessment of the dysregulated lncRNAs and mRNAs by reverse transcription-quantitative PCR in vitro. (A) TLR4 protein expression in CTL FHC cells, and FHC cells treated with LPS. Relative mRNA expression levels of (B) IL-6, (C) IL-1 $\beta$, (D) TM4SF20, (E) SLC5A12 and (F) LRRC19 in the CTL and NEC groups. Relative lncRNA expression levels of (G) NONHSAT022134.2, (H) NONHSAT076868.2, (I) NONHSAT159417.1, (J) NONHSAT094312.2 and (K) NONHSAT142596.2 in the CTL and NEC groups. Data are presented as mean \pm standard deviation. ${ }^{*} \mathrm{P}<0.05,{ }^{* *} \mathrm{P}<0.01$, ${ }^{* * *} \mathrm{P}<0.001$. CTL, control; IncRNA, long non-coding RNA; IL, interleukin; TM4SF20, Transmembrane 4 L six family member 20; SLC5A12, solute carrier family 5 member 12; LRRC19, leucine rich repeat containing 19; NEC, necrotizing enterocolitis. 
A

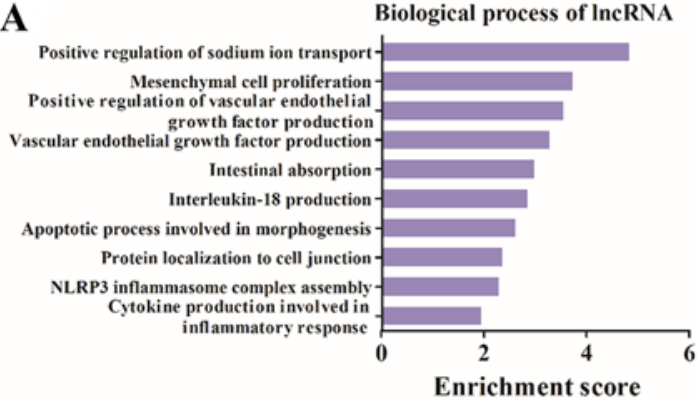

C

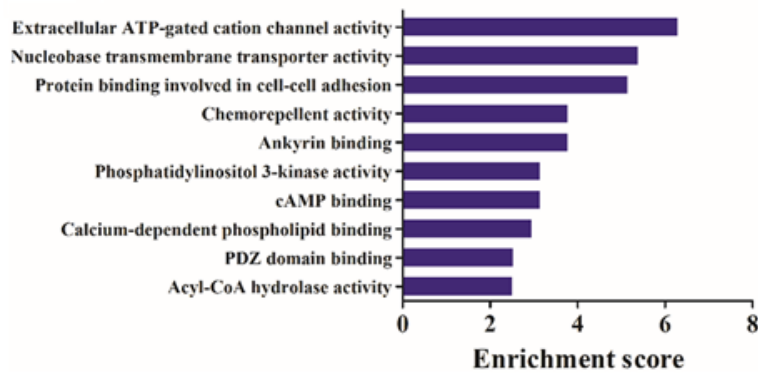

B

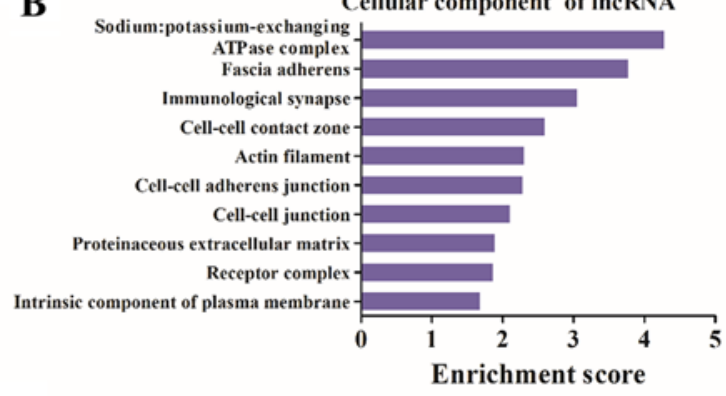

D

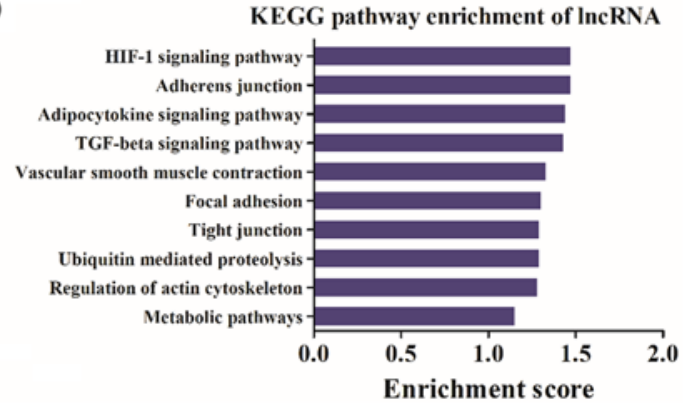

Figure 4. Gene Ontology and pathway analysis predicted the potential function of the differentially expressed lncRNA host genes. (A) Biological process category. (B) Cellular component category. (C) Molecular function category. (D) Canonical signaling pathways. lncRNA, long non-coding RNA.

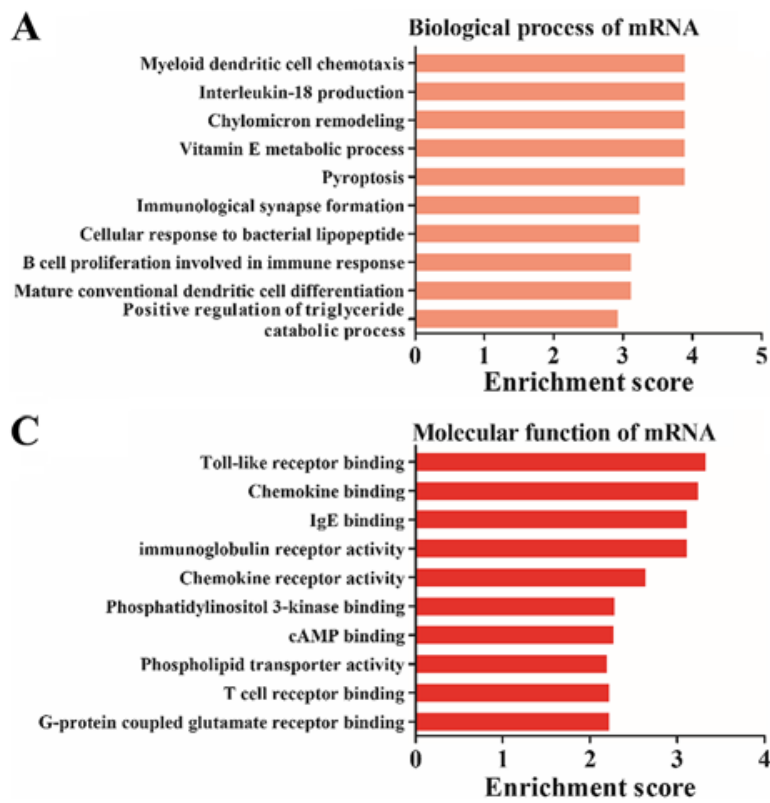

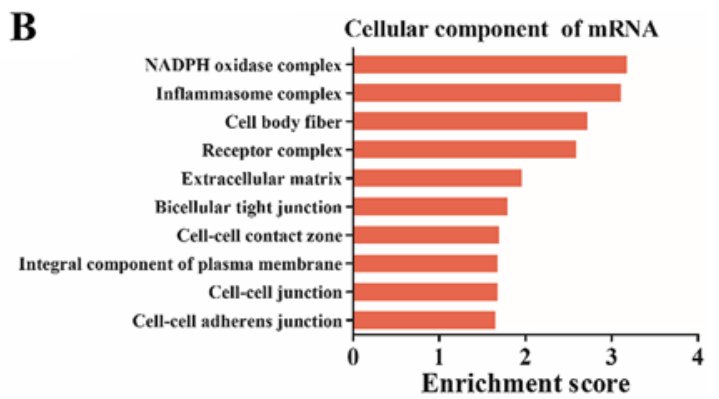

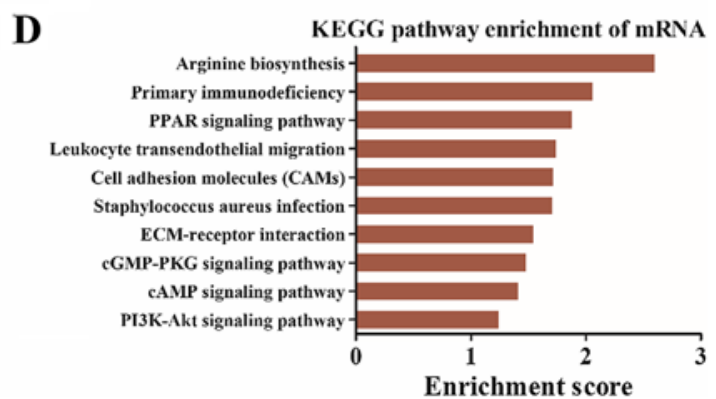

Figure 5. Gene Ontology and pathway analysis predicted the potential function of the differentially expressed mRNA. (A) Biological process category. (B) Cellular component category. (C) Molecular function category. (D) Canonical signaling pathways.

differentially expressed lncRNA host genes were involved in 'TGF- $\beta$ signaling pathway', 'hypoxia-inducible factor 1 (HIF-1) signaling pathway' and 'tight junction and regulation of actin cytoskeleton' (Fig. 4D).

Differentially expressed mRNAs are involved in TLR binding and the PI3K-Akt signaling pathway. GO and KEGG analysis were also performed for the differentially expressed mRNAs, which were significantly enriched in 'myeloid dendritic cell chemotaxis' (biological process; Fig. 5A), 'inflammasome complex' (cellular component; Fig. 5B) and 'TLR binding' (molecular function; Fig. 5C). Furthermore, KEGG pathway analysis results demonstrated that the differentially expressed mRNAs were enriched in 'peroxisome proliferator-activated receptors (PPAR) signaling pathway', 'leukocyte transendothelial migration', 'extracellular matrix-receptor interaction' and 'PI3K-Akt signaling pathway' (Fig. 5D).

IncRNA-mRNA network reveals a potential mechanism for $N E C$. The lncRNA-mRNA co-expression network results suggested that IncRNAs had a complex interaction with mRNAs (Fig. 6). A total of 80 associations were identified 


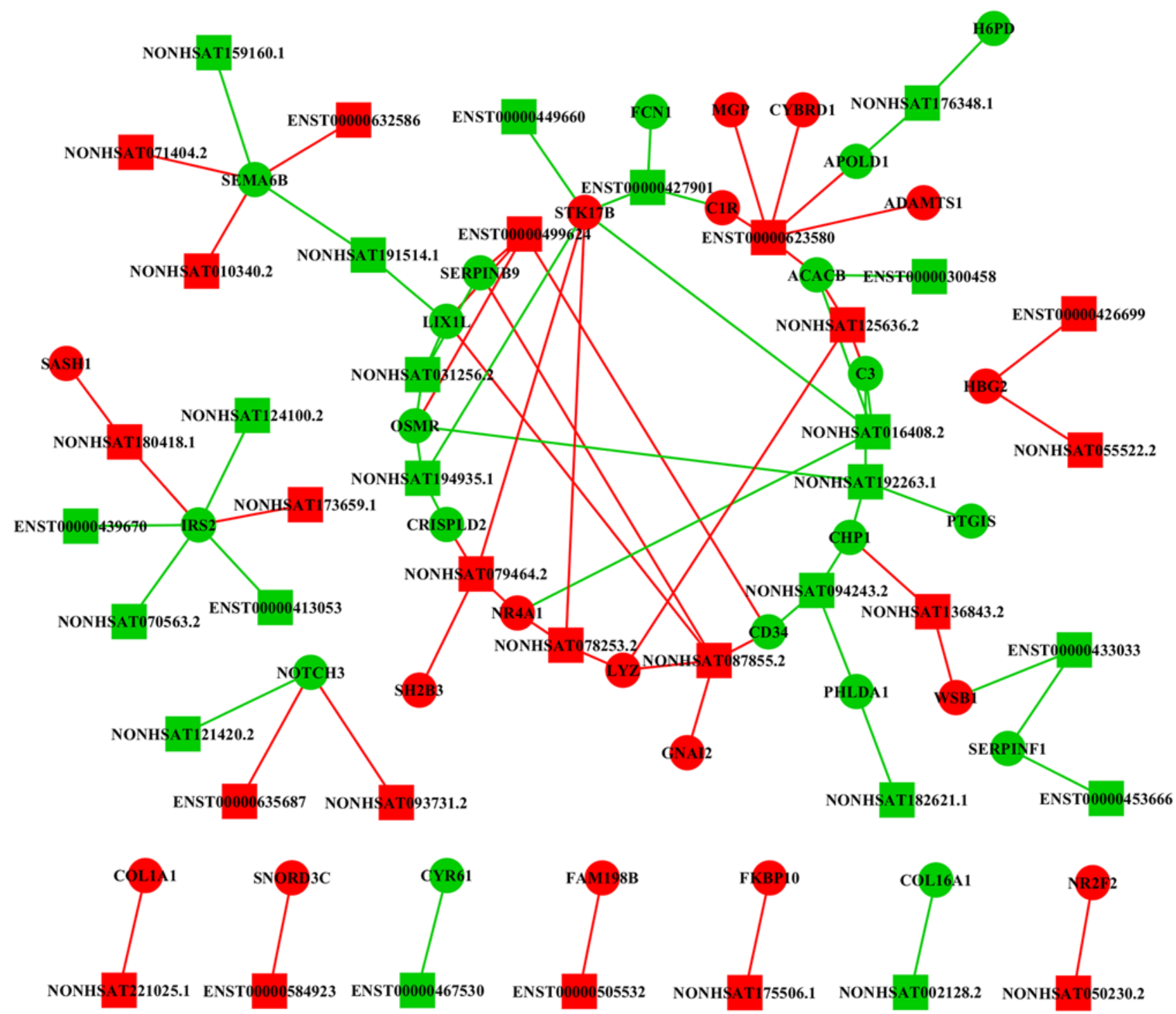

Figure 6. Construction of the lncRNA-mRNA co-expression network. Circles represent mRNAs and squares represent lncRNAs. Upregulation and downregulation are presented in red and green, respectively. Red and green lines represent positive and negative associations, respectively. lncRNA, long non-coding RNA.

among the 44 lncRNAs and 38 mRNAs. Moreover, it was identified that lncRNAs ENST00000623580, NONHSAT180418.1, NONHSAT125636.2 and NONHSAT087855.2 interacted with mRNAs that were closely associated with NEC. Therefore, the present results suggested that these lncRNAs may serve important roles in the pathogenesis of NEC.

\section{Discussion}

To the best of our knowledge, no previous studies have examined lncRNAs associated with NEC, and there is no functional evidence from studies investigating lncRNAs with NEC and intestinal function. The present study analyzed the differentially expressed lncRNAs and mRNAs in ileal samples obtained from premature neonates with NEC. Furthermore, an in vitro cell model of NEC was investigated. The aims of the present study were to predict the potential functions of the differentially expressed lncRNAs and mRNAs, and to identify a potential mechanism for NEC via IncRNA-mRNA network analyses. While the pathophysiological mechanism of NEC remains unknown, previous studies have reported that the expression of TLR4 is upregulated in NEC $(20,21)$. The activation of TLR4 on intestinal epithelial cells induces a significant proinflammatory response (22). Furthermore, TLR4 activation also results in increased intestinal necrosis and apoptosis, which is the main feature of NEC (19). In addition, it has been shown that LPS-induced reactive oxygen species accumulation is involved in NF- $\mathrm{NB}$ activation and subsequent release of proinflammation cytokines, including IL- 6 and IL-1 $\beta$, in the pathogenesis of NEC $(23,24)$. A previous study revealed that excessive inflammation of the immature intestine predisposes premature infants to NEC (25). Therefore, elevated levels of IL- 6 and IL-1 $\beta$ may be used as an index of successful NEC cell model construction (26-28). The present results suggested that the expression levels of IL-1 $\beta$, IL- 6 and TLR4 in FHCs were significantly increased following LPS exposure, thus indicating that this was a reliable cell model for studying NEC in vitro. 
GO and KEGG analyses were performed to identify potential mechanisms underlying NEC. The GO analysis results demonstrated that both the differentially expressed lncRNA host genes and mRNAs were associated with inflammation, which is a typical feature of NEC (29). Moreover, it was identified that TLR binding was a highly enriched molecular function, thus indicating the importance of the TLR in NEC. The results of KEGG pathway analysis identified pathways that were mainly associated with the progression of NEC. The PPAR pathway has been shown to affect mucosal immunity, commensal homeostasis and intestinal inflammation (30). Moreover, PPAR $\alpha$ downregulation in mice contributes to an imbalance of intestinal symbiosis, resulting in enhanced susceptibility to intestinal inflammation and increased production of inflammatory cytokines (30). Previous studies have shown that the PI3K-Akt signaling pathway is altered in an experimental NEC animal model $(31,32)$. Furthermore, berberine inhibits apoptosis and inflammation via the PI3K/AKT signaling pathway to attenuate the progression of NEC (32). Macrophages exhibited increased Smad7 expression in areas with high bacterial load and severe tissue damage (33). In addition, elevated Smad7 expression inhibits TGF- $\beta$ signaling in intestinal macrophages, and promotes NF- $\mathrm{kB}$ activation and cytokine production during NEC (33). The PI3K-Akt signaling pathway has also been reported to regulate HIF-1 to protect against intestinal injury during NEC (34).

The IncRNA-mRNA network constructed in the present study revealed the IncRNAs and mRNAs that may be potentially involved in NEC. IncRNA ENST00000623580 was identified to interact with 6 mRNAs, including ADAM metallopeptidase with thrombospondin type 1 motif 1 (ADAMTS1), complement $\mathrm{C} 1 \mathrm{R}$, apolipoprotein $\mathrm{L}$ domain containing 1 (APOLD1), cytochrome B reductase 1 (CYBRD1), acetyl-CoA carboxylase $\beta$ (ACACB) and matrix Gla protein (MGP). Among them, ADAMTS1 is involved in various inflammatory processes and is required for maintaining organ function, including the intestine $(35,36)$. Moreover, C1R encodes several members of the peptidase S1 protein family, which are proteins that serve as a proteolytic subunit in the complement system $\mathrm{C} 1$ complex (37). The complement system acts as a mediator in the innate immune response by triggering phagocytosis, inflammation and rupture of bacterial cell walls, which may affect the progression of NEC (38). APOLD1 is an endothelial cell early response protein that affects vascular function and regulates endothelial cell signaling, which are closely associated with the NEC process (39). As a member of the cytochrome family, CYBRD1 encodes an iron-regulated protein and exhibits ferric reductase activity (40). Furthermore, CYBRD1 has been demonstrated to serve a key role in dietary iron absorption (41). It has been shown that NEC may affect the expression of CYBRD1 in the duodenal brush border membrane and contributes to the severe anemia associated with NEC (42). ACACB is currently considered to be one of the potential targets for regulating human disease in diabetes, obesity and cancer (43). It was demonstrated that continuous oxidation of fatty acid in ACACB knockout mice can lead to high insulin sensitivity (44). Moreover, a change in tissue ACACB levels via transcriptional regulation may be important in the affected intestine in NEC (45). MGP encodes a member of the osteocalcin/matrix Gla family of proteins (46). The encoded vitamin K-dependent protein is secreted by chondrocytes and vascular smooth muscle cells, and functions as a physiological inhibitor of ectopic tissue calcification (46). Furthermore, the present results suggested that lncRNA ENST00000623580 may be involved in the pathogenesis of NEC by regulating the expression of the aforementioned mRNAs.

SAM and SH3 Domain Containing 1 (SASH1) was identified to be associated with lncRNA NONHSAT180418.1. SASH1 is expressed on all microvascular beds and serves as a scaffold molecule to bind TBGF- $\beta$-activated kinase 1 (TAK1), TNF receptor associated factor 6 (TRAF6), IkB kinase $\alpha$ and IкB kinase $\beta$ (47). This interaction promotes the ubiquitination of TAK1 and TRAF6, and promotes LPS-induced activation of NF- $\mathrm{KB}, \mathrm{p} 38$ and JNK, which leads to increased expression of proinflammatory cytokines and increased endothelial migration (47). Moreover, SASH1 has been shown to be involved in downstream signaling of TLR4 and to activate early endothelial responses to receptor activation (48). Therefore, IncRNA NONHSAT180418.1 was hypothesized to regulate the expression of SASH1 to affect TLR4 and the progression of NEC. In addition, lncRNAs NONHSAT125636.2 and NONHSAT087855.2 were both associated with LYZ. LYZ encodes human lysozyme, whose natural substrate is the bacterial cell wall component peptidoglycan (49). It was reported that increasing the expression of LYZ may control the microbial load in the intestine (50). Therefore, lncRNA NONHSAT125636.2 and NONHSAT087855.2 may exert protective effects in NEC. It has been revealed that lncRNAs can function as competing endogenous RNAs to indirectly regulate the expression levels of target genes via sponging microRNAs, and they are implicated in numerous biological process $(51,52)$. Thus, further studies on the IncRNA-associated competing endogenous RNA regulatory network may be useful for understanding the pathogenesis of NEC.

In conclusion, the present study identified differentially expressed lncRNAs and mRNAs between NEC and adjacent intestinal tissues by NGS. A number of these genes were randomly selected and assessed by RT-qPCR in vitro. GO and KEGG analyses were performed, and indicated that lncRNAs ENST00000623580, NONHSAT180418.1, NONHSAT125636.2 and NONHSAT087855.2 may have bioactive effects in NEC. Therefore, the present study may provide novel insights into the pathological mechanisms underlying NEC. However, further functional investigation is required to confirm these results.

\section{Acknowledgements}

Not applicable.

\section{Funding}

The present study was supported by grants from the Wuxi Key MedicalDisciplines(grantno.ZDXK12),theMedicalInnovation Team of Jiangsu Province (grant no. CXTDB2017016), the Wuxi Medical Development Discipline (grant no. FZXK001), the Wuxi Young Medical Talents (grant nos. QNRC094 and QNRC089), the Wuxi Hospital Management Centre Key Project (grant no. YGZXZ1513) and the Wuxi Hospital Management 
Centre Joint Research Project (grant no. YGZXL1319). The study was also support by the Young Project of Wuxi Health and Family Planning Commission (grant no. Q201815), the National Natural Science Foundation of China (grant nos. 81741089 and 81971427), the Jiangsu Maternal and Child Health Research Project (grant no. F2018525), the National Natural Science Foundation for Youth Scholars of China (grant nos. 81501296, 81901512 and 81901517) and the Nanjing Medical Science and Technological Development Program (grant no. YKK17176).

\section{Availability of data and materials}

The datasets used and/or analyzed during the current study are available from the corresponding author on reasonable request.

\section{Authors' contributions}

WC, XY, TT, RY, XW, ZY and YL collected and analyzed the data. LZ and SH designed the study and wrote the manuscript. All authors read and approved the final manuscript.

\section{Ethics approval and consent to participate}

The study protocol was approved by the Institutional Review Board of The Affiliated Wuxi Children's Hospital of Nanjing Medical University (approval no. 2017-EYLL-115). Informed consent was obtained from the parents or guardians of all patients.

\section{Patient consent for publication}

Informed consent was obtained from the parents or guardians of all patients.

\section{Competing interests}

The authors declare that they have no competing interests.

\section{References}

1. Fitzgibbons SC, Ching Y, Yu D, Carpenter J, Kenny M, Weldon C, Lillehei C, Valim C, Horbar JD and Jaksic T: Mortality of necrotizing enterocolitis expressed by birth weight categories. J Pediatr Surg 44: 1072-1076, 2009.

2. Kastenberg ZJ, Lee HC, Profit J, Gould JB and Sylvester KG: Effect of deregionalized care on mortality in very low-birth-weight infants with necrotizing enterocolitis. JAMA Pediatr 169: 26-32, 2015.

3. Dukleska K, Devin CL, Martin AE, Miller JM, Sullivan KM, Levy C, Prestowitz S, Flathers K, Vinocur CD and Berman L: Necrotizing enterocolitis totalis: High mortality in the absence of an aggressive surgical approach. Surgery 165: 1176-1181, 2019.

4. Lee JS and Polin RA: Treatment and prevention of necrotizing enterocolitis. Semin Neonatol 8: 449-459, 2003.

5. Shulhan J, Dicken B, Hartling L and Larsen BM: Current Knowledge of Necrotizing Enterocolitis in Preterm Infants and the Impact of Different Types of Enteral Nutrition Products. Adv Nutr 8: 80-91, 2017.

6. Kim ED and Sung S: Long noncoding RNA: Unveiling hidden layer of gene regulatory networks. Trends Plant Sci 17: 16-21, 2012.

7. Goff LA and Rinn JL: Linking RNA biology to lncRNAs. Genome Res 25: 1456-1465, 2015.

8. Han Y, Hong Y, Li L, Li T, Zhang Z, Wang J, Xia H, Tang Y, Shi Z, Han X, et al: A Transcriptome-Level Study Identifies Changing Expression Profiles for Ossification of the Ligamentum Flavum of the Spine. Mol Ther Nucleic Acids 12: 872-883, 2018.
9. Kim YJ,Hwang KC, Kim SW and Lee YC: Potential miRNA-target interactions for the screening of gastric carcinoma development in gastric adenoma/dysplasia. Int J Med Sci 15: 610-616, 2018.

10. Xiao L, Rao JN, Cao S, Liu L, Chung HK, Zhang Y, Zhang J, Liu Y, Gorospe $M$ and Wang JY: Long noncoding RNA SPRY4-IT1 regulates intestinal epithelial barrier function by modulating the expression levels of tight junction proteins. Mol Biol Cell 27: 617-626, 2016.

11. Geng H, Bu HF, Liu F, Wu L, Pfeifer K, Chou PM, Wang X, Sun J, Lu L, Pandey A, et al: In Inflamed Intestinal Tissues and Epithelial Cells, Interleukin 22 Signaling Increases Expression of H19 Long Noncoding RNA, Which Promotes Mucosal Regeneration. Gastroenterology 155: 144-155, 2018.

12. Bell MJ, Ternberg JL, Feigin RD, Keating JP, Marshall R, Barton L and Brotherton T: Neonatal necrotizing enterocolitis. Therapeutic decisions based upon clinical staging. Ann Surg 187: $1-7,1978$.

13. Mortazavi A, Williams BA, McCue K, Schaeffer L and Wold B: Mapping and quantifying mammalian transcriptomes by RNA-Seq. Nat Methods 5: 621-628, 2008.

14. Liu M, Song S, Li H, Jiang X, Yin P, Wan C, Liu X, Liu F and $\mathrm{Xu}$ J: The protective effect of caffeic acid against inflammation injury of primary bovine mammary epithelial cells induced by lipopolysaccharide. J Dairy Sci 97: 2856-2865, 2014.

15. Livak KJ and Schmittgen TD: Analysis of relative gene expression data using real-time quantitative PCR and the 2(-Delta Delta C(T)) Method. Methods 25: 402-408, 2001.

16. Robinson MD, McCarthy DJ and Smyth GK: edgeR: A Bioconductor package for differential expression analysis of digital gene expression data. Bioinformatics 26: 139-140, 2010.

17. Yazji I, Sodhi CP, Lee EK, Good M, Egan CE, Afrazi A, Neal MD, Jia H, Lin J, Ma C, et al: Endothelial TLR4 activation impairs intestinal microcirculatory perfusion in necrotizing enterocolitis via eNOS-NO-nitrite signaling. Proc Natl Acad Sci USA 110: 9451-9456, 2013.

18. Pang Y, Du X, Xu X, Wang M and Li Z: Monocyte activation and inflammation can exacerbate Treg/Th17 imbalance in infants with neonatal necrotizing enterocolitis. Int Immunopharmacol 59: 354-360, 2018.

19. Good M, Siggers RH, Sodhi CP, Afrazi A, Alkhudari F, Egan CE, Neal MD, Yazji I, Jia H, Lin J, et al: Amniotic fluid inhibits Toll-like receptor 4 signaling in the fetal and neonatal intestinal epithelium. Proc Natl Acad Sci USA 109: 11330-11335, 2012.

20. Jilling T, Simon D, Lu J, Meng FJ, Li D, Schy R, Thomson RB, Soliman A, Arditi M and Caplan MS: The roles of bacteria and TLR4 in rat and murine models of necrotizing enterocolitis. J Immunol 177: 3273-3282, 2006.

21. Sharma R, Tepas JJ III, Hudak ML, Mollitt DL, Wludyka PS, Teng RJ and Premachandra BR: Neonatal gut barrier and multiple organ failure: Role of endotoxin and proinflammatory cytokines in sepsis and necrotizing enterocolitis. J Pediatr Surg 42: 454-461, 2007.

22. Bonora M, Wieckowsk MR, Chinopoulos C, Kepp O, Kroemer G, Galluzzi L and Pinton P: Molecular mechanisms of cell death: Central implication of ATP synthase in mitochondrial permeability transition. Oncogene 34: 1608, 2015.

23. Zhang L, Fan J, He J, Chen W, Jin W, Zhu Y, Sun H, Li Y, Shi Y, Jing Y, et al: Regulation of ROS-NF- $\kappa \mathrm{B}$ axis by tuna backbone derived peptide ameliorates inflammation in necrotizing enterocolitis. J Cell Physiol 234: 14330-14338, 2019.

24. Good M, Sodhi CP, Egan CE, Afrazi A, Jia H, Yamaguchi Y, Lu P, Branca MF, Ma C, Prindle T Jr, et al: Breast milk protects against the development of necrotizing enterocolitis through inhibition of Toll-like receptor 4 in the intestinal epithelium via activation of the epidermal growth factor receptor. Mucosal Immunol 8: 1166-1179, 2015.

25. Wijendran V, Brenna JT, Wang DH, Zhu W, Meng D, Ganguli K, Kothapalli KS, Requena P, Innis S and Walker WA: Long-chain polyunsaturated fatty acids attenuate the IL-1 $\beta$-induced proinflammatory response in human fetal intestinal epithelial cells. Pediatr Res 78: 626-633, 2015.

26. Feng Z, Zhou H, Ma S, Guan X, Chen L, Huang J, Gui H, Miao X, Yu S, Wang JH and Wang J: FTY720 attenuates intestinal injury and suppresses inflammation in experimental necrotizing enterocolitis via modulating CXCL5/CXCR2 axis. Biochem Biophys Res Commun 505: 1032-1037, 2018.

27. Chan KY, Leung FW, Lam HS, Tam YH, To KF, Cheung HM, Leung KT, Poon TC, Lee KH, Li K, et al: Immunoregulatory protein profiles of necrotizing enterocolitis versus spontaneous intestinal perforation in preterm infants. PLoS One 7: e36977, 2012. 
28. Sheng Q, Lv Z, Cai W, Song H, Qian L, Mu H, Shi J and Wang X: Human $\beta$-defensin-3 promotes intestinal epithelial cell migration and reduces the development of necrotizing enterocolitis in a neonatal rat model. Pediatr Res 76: 269-279, 2014.

29. Kandasamy J, Huda S, Ambalavanan $N$ and Jilling T: Inflammatory signals that regulate intestinal epithelial renewal, differentiation, migration and cell death: Implications for necrotizing enterocolitis. Pathophysiology 21: 67-80, 2014.

30. Manoharan I, Suryawanshi A, Hong Y, Ranganathan P, Shanmugam A, Ahmad S, Swafford D, Manicassamy B, Ramesh G, Koni PA, et al: Homeostatic PPARalpha Signaling Limits Inflammatory Responses to Commensal Microbiota in the Intestine. J Immunol 196: 4739-4749, 2016.

31. Shiou SR, Yu Y, Chen S, Ciancio MJ, Petrof EO, Sun J and Claud EC: Erythropoietin protects intestinal epithelial barrier function and lowers the incidence of experimental neonatal necrotizing enterocolitis. J Biol Chem 286: 12123-12132, 2011

32. Fang C, Xie L, Liu C, Fu C, Ye W, Liu H and Zhang B: Berberine ameliorates neonatal necrotizing enterocolitis by activating the phosphoinositide 3-kinase/protein kinase B signaling pathway. Exp Ther Med 15: 3530-3536, 2018.

33. MohanKumar K, Namachivayam K, Chapalamadugu KC, Garzon SA, Premkumar MH, Tipparaju SM and Maheshwari A: Smad7 interrupts TGF- $\beta$ signaling in intestinal macrophages and promotes inflammatory activation of these cells during necrotizing enterocolitis. Pediatr Res 79: 951-961, 2016.

34. Baregamian N, Rychahou PG, Hawkins HK, Evers BM and Chung DH: Phosphatidylinositol 3-kinase pathway regulates hypoxia-inducible factor-1 to protect from intestinal injury during necrotizing enterocolitis. Surgery 142: 295-302, 2007.

35. Günther W, Skaftnesmo KO, Arnold H, Bjerkvig R and Terzis AJ: Distribution patterns of the anti-angiogenic protein ADAMTS-1 during rat development. Acta Histochem 107: 121-131, 2005.

36. Hummitzsch L, Zitta K, Berndt R, Wong YL, Rusch R, Hess K, Wedel T, Gruenewald M, Cremer J, Steinfath M, et al: Remote ischemic preconditioning attenuates intestinal mucosal damage: Insight from a rat model of ischemia-reperfusion injury. J Trans Med 17: 136, 2019.

37. Kardos J, Harmat V, Palló A, Barabás O, Szilágyi K, Gráf L, Náray-Szabó G, Goto Y, Závodszky P and Gál P: Revisiting the mechanism of the autoactivation of the complement protease $\mathrm{C} 1 \mathrm{r}$ in the $\mathrm{C} 1$ complex: Structure of the active catalytic region of $\mathrm{Clr}$. Mol Immunol 45: 1752-1760, 2008.

38. Kobayashi H, Albarracin L, Sato N, Kanmani P, Kober AK, Ikeda-Ohtsubo W, Suda Y, Nochi T, Aso H, Makino S, et al: Modulation of porcine intestinal epitheliocytes immunetranscriptome response by Lactobacillus jensenii TL2937. Benef Microbes 7: 769-782, 2016.

39. Regard JB, Scheek S, Borbiev T, Lanahan AA, Schneider A, Demetriades AM, Hiemisch H, Barnes CA, Verin AD and Worley PF: Verge: a novel vascular early response gene. J Neurosci 24: 4092-4103, 2004
40. Oakhill JS, Marritt SJ, Gareta EG, Cammack R and McKie AT: Functional characterization of human duodenal cytochrome B (Cybrd1): Redox properties in relation to iron and ascorbate metabolism. Biochim Biophys Acta 1777: 260-268, 2008.

41. Schlottmann F, Vera-Aviles M and Latunde-Dada GO: Duodenal cytochrome B (Cybrd1) ferric reductase functional studies in cells. Metallomics 9: 1389-1393, 2017.

42. Patel RM, Knezevic A, Shenvi N, Hinkes M, Keene S, Roback JD, Easley KA and Josephson CD: Association of Red Blood Cell Transfusion, Anemia, and Necrotizing Enterocolitis in Very Low-Birth-Weight Infants. JAMA 315: 889-897, 2016.

43. Glorieux C, Zamocky M, Sandoval JM, Verrax J and Calderon PB: Regulation of catalase expression in healthy and cancerous cells. Free Radic Biol Med 87: 84-97, 2015.

44. Ma L, Mondal AK, Murea M, Sharma NK, Tönjes A, Langberg KA, Das SK, Franks PW, Kovacs P, Antinozzi PA, et al: The effect of ACACB cis-variants on gene expression and metabolic traits. PLoS One 6: e23860, 2011.

45. Chen G, Li Y, Su Y, Zhou L, Zhang H, Shen Q, Du C, Li H, Wen Z, Xia Y, et al: Identification of candidate genes for necrotizing enterocolitis based on microarray data. Gene 661: 152-159, 2018.

46. Caiado H, Conceição N, Tiago D, Marreiros A, Vicente S, Enriquez JL, Vaz AM, Antunes A, Guerreiro H, Caldeira P, et al: Evaluation of MGP gene expression in colorectal cancer. Gene 723: 144120, 2020.

47. Dauphinee SM, Clayton A, Hussainkhel A, Yang C, Park YJ, Fuller ME, Blonder J, Veenstra TD and Karsan A: SASH1 is a scaffold molecule in endothelial TLR4 signaling. J Immunol 191: 892-901, 2013.

48. Coulombe P, Paliouras GN, Clayton A, Hussainkhel A, Fuller M, Jovanovic V, Dauphinee S, Umlandt P, Xiang P, Kyle AH, et al: Endothelial Sash1 Is Required for Lung Maturation through Nitric Oxide Signaling. Cell Rep 27: 1769-1780.e1764, 2019

49. Um SH, Kim JS, Kim K, Kim N, Cho HS and Ha NC: Structural basis for the inhibition of human lysozyme by PliC from Brucella abortus. Biochemistry 52: 9385-9393, 2013.

50. Singh SB, Wilson M, Ritz N and Lin HC: Autophagy Genes of Host Responds to Disruption of Gut Microbial Community by Antibiotics. Dig Dis Sci 62: 1486-1497, 2017.

51. Kallen AN, Zhou XB, Xu J, Qiao C, Ma J, Yan L, Lu L, Liu C, Yi JS, Zhang H, et al: The imprinted H19 lncRNA antagonizes let-7 microRNAs. Mol Cell 52: 101-112, 2013.

52. Fan Z, Gao S, Chen Y, Xu B, Yu C, Yue M and Tan X: Integrative analysis of competing endogenous RNA networks reveals the functional lncRNAs in heart failure. J Cell Mol Med 22: 4818-4829, 2018.

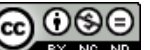

This work is licensed under a Creative Commons Attribution-NonCommercial-NoDerivatives 4.0 International (CC BY-NC-ND 4.0) License. 